Supplement of Biogeosciences, 19, 743-762, 2022

https://doi.org/10.5194/bg-19-743-2022-supplement

(c) Author(s) 2022. CC BY 4.0 License.

(c) (i)

Supplement of

\title{
Late Neogene evolution of modern deep-dwelling plankton
}

Flavia Boscolo-Galazzo et al.

Correspondence to: Flavia Boscolo-Galazzo (flavia.boscologalazzo@uib.no)

The copyright of individual parts of the supplement might differ from the article licence. 
Supplementary Figures
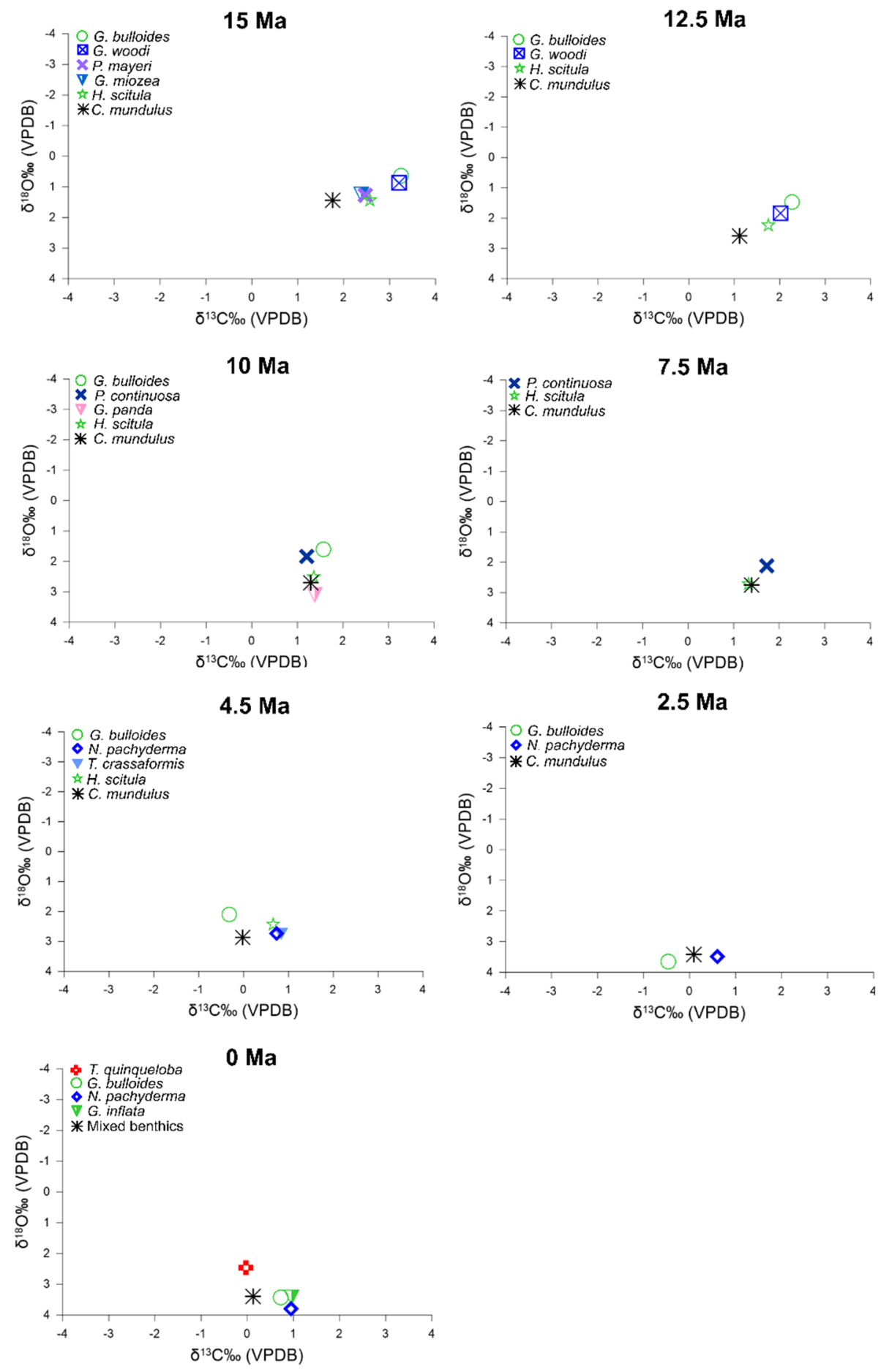

Figure S1. Foraminiferal Oxygen and Carbon stable isotope plots for Site 1138. Colored symbols indicate planktonic foraminifera, the black asterisk indicates benthic foraminifera. 


\section{$15 \mathrm{Ma}$}
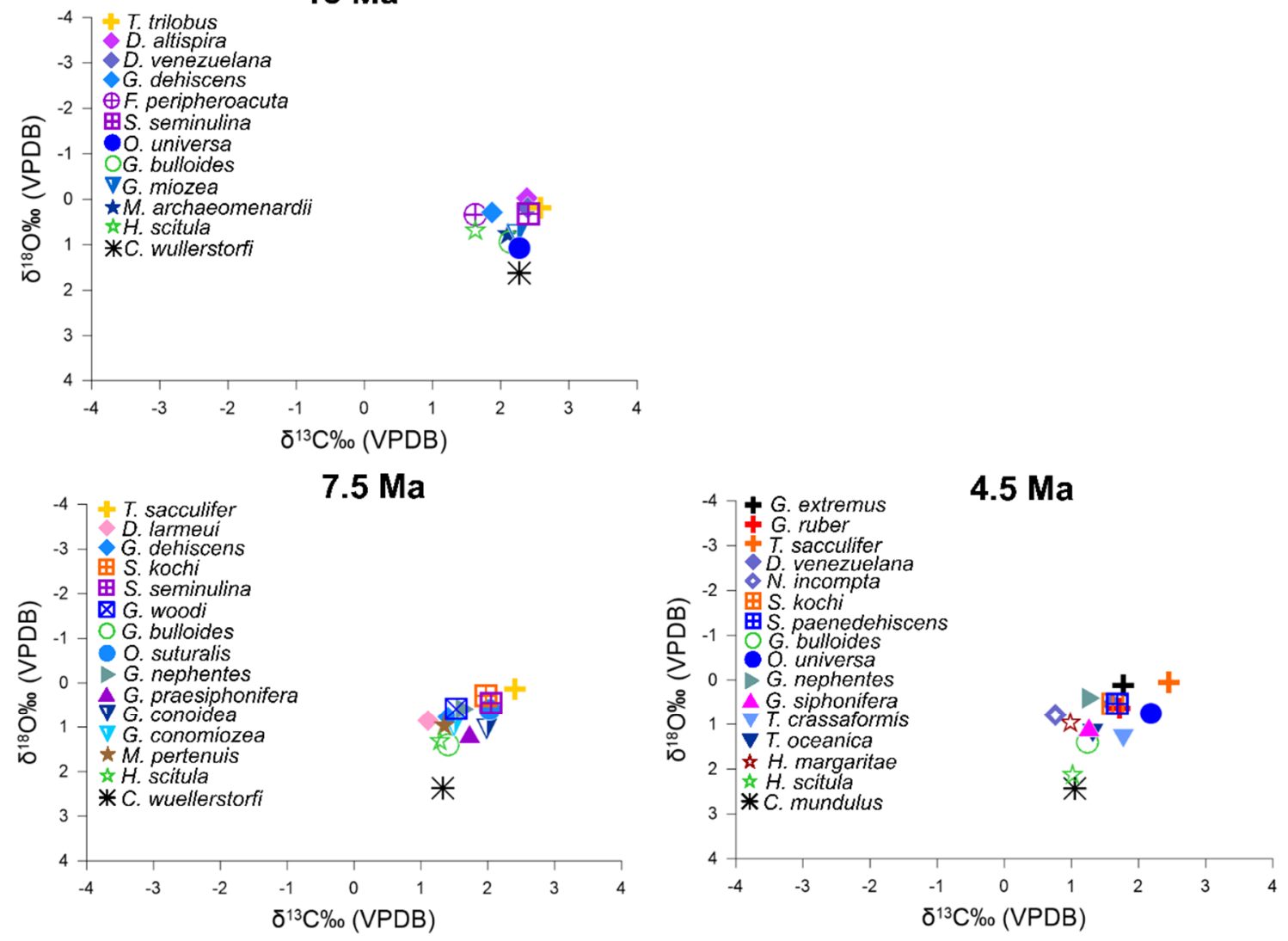

$2.5 \mathrm{Ma}$
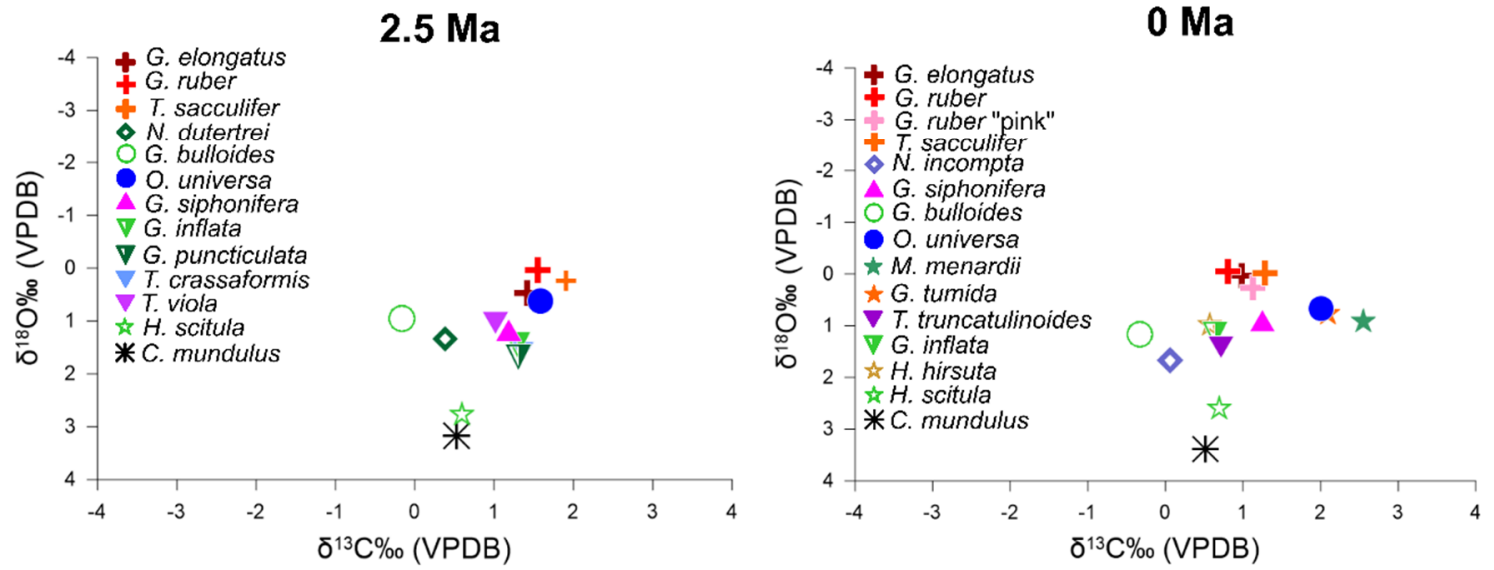

Figure S2. Foraminiferal Oxygen and Carbon stable isotope plots for Site 516. Colored symbols indicate planktonic foraminifera, the black asterisk indicates benthic foraminifera. 

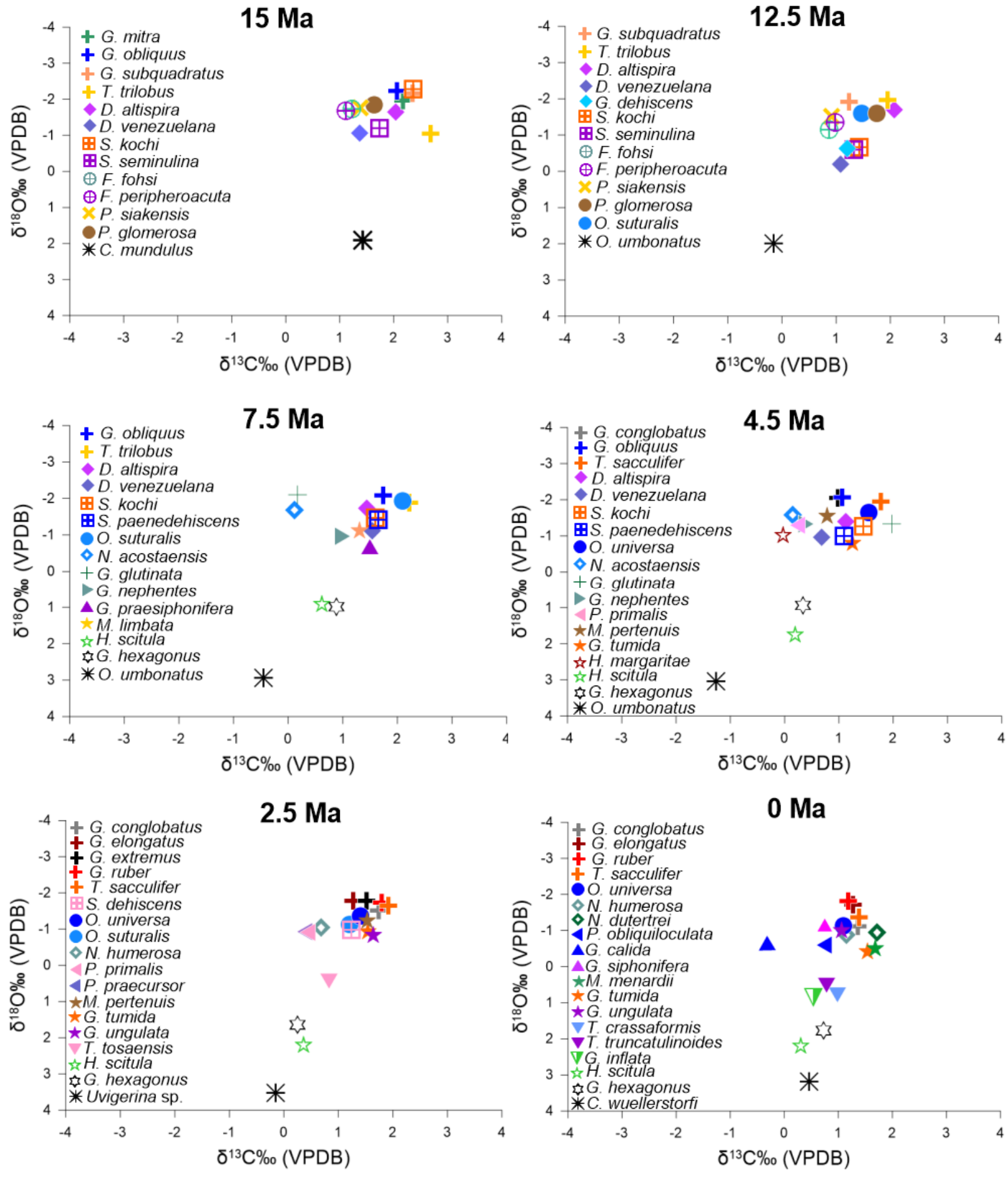

Figure S3. Foraminiferal Oxygen and Carbon stable isotope plots for Site 242. Colored symbols indicate planktonic foraminifera, the black asterisk indicates benthic foraminifera. 

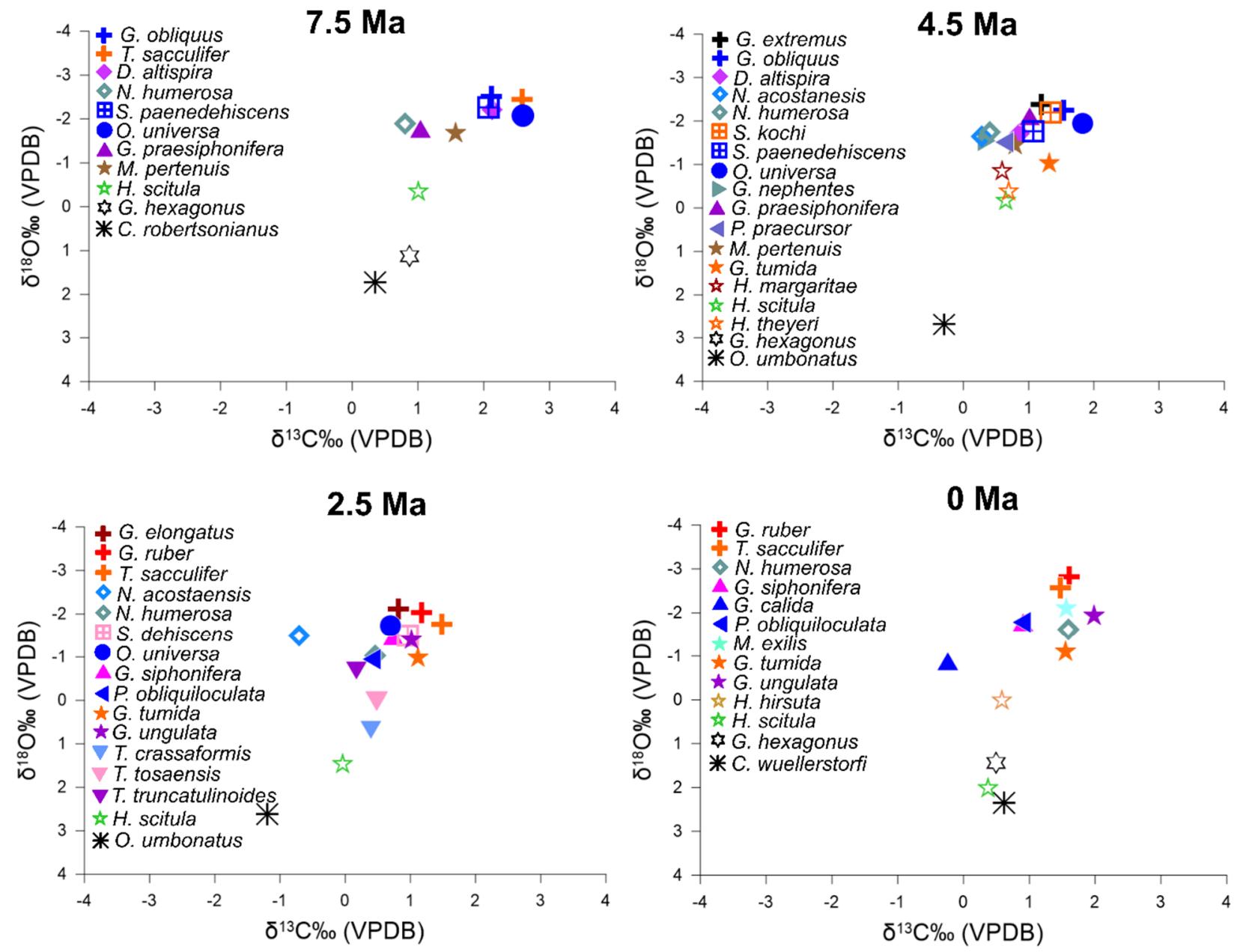

Figure S4. Foraminiferal Oxygen and Carbon stable isotope plots for Site U1482. Colored symbols indicate planktonic foraminifera, the black asterisk indicates benthic foraminifera. 
$15 \mathrm{Ma}$
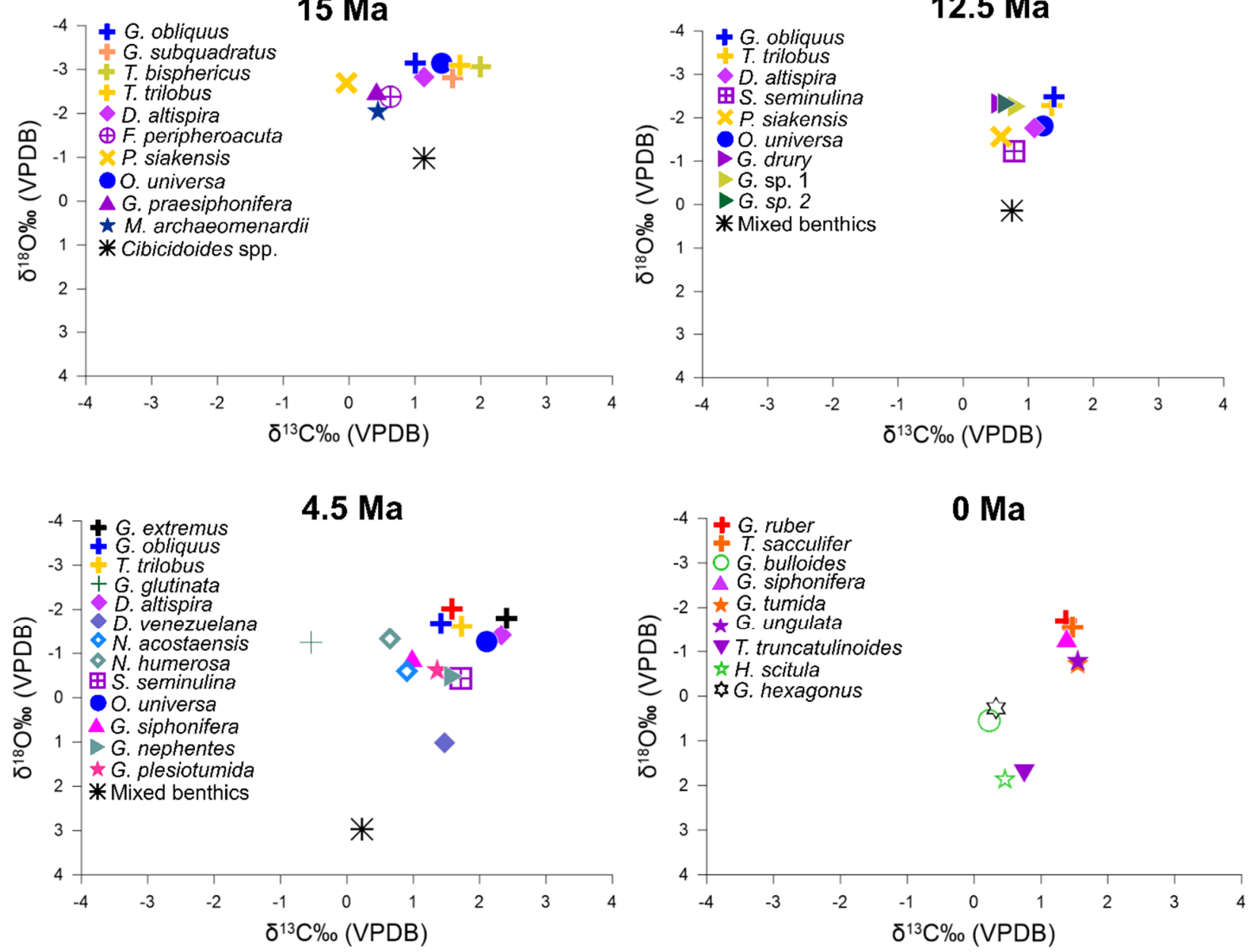

Figure S5. Foraminiferal Oxygen and Carbon stable isotope plots for Tanzania-GLOW sites. Colored symbols indicate planktonic foraminifera, the black asterisk indicates benthic foraminifera. 
$15 \mathrm{Ma}$
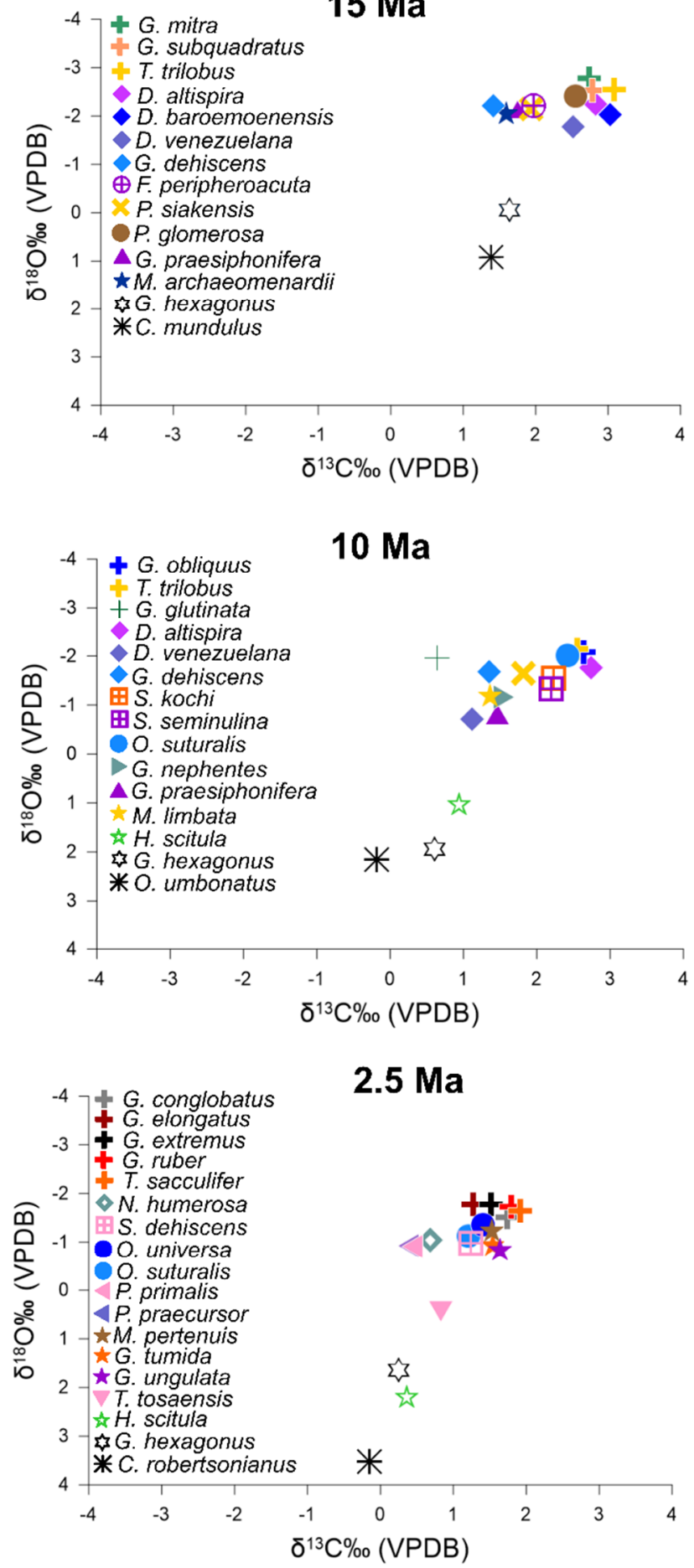
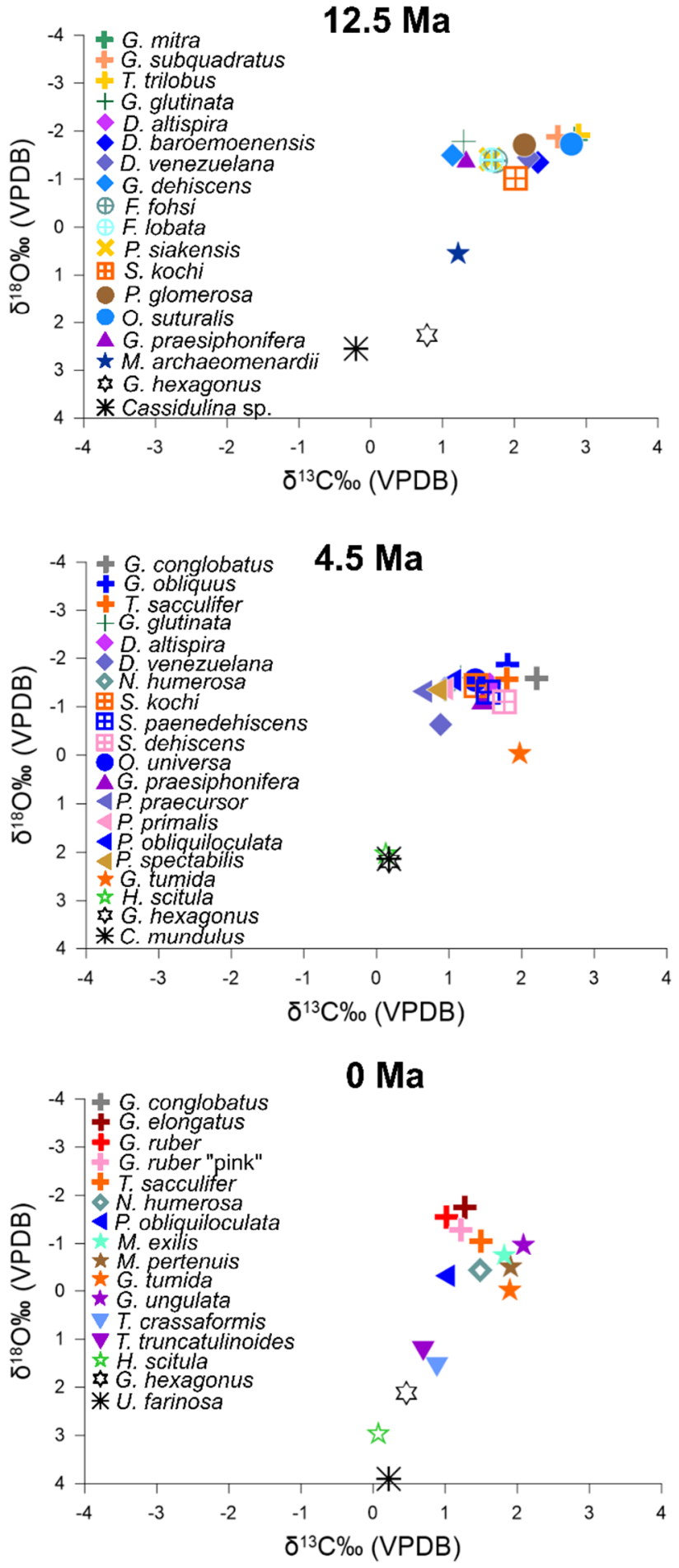

Figure S6. Foraminiferal Oxygen and Carbon stable isotope plots for Site 871-872. Colored symbols indicate planktonic foraminifera, the black asterisk indicates benthic foraminifera. 

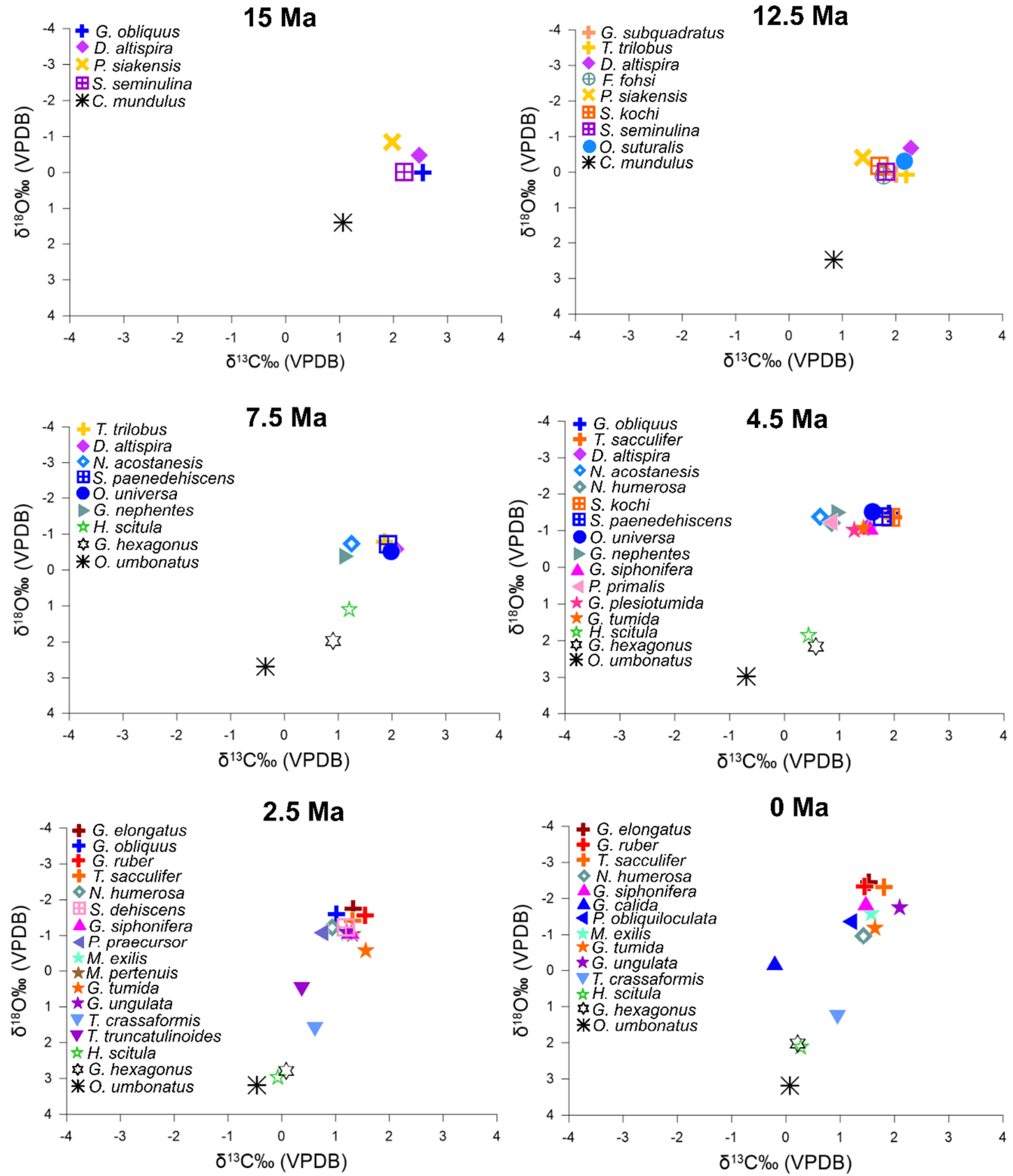

Figure S7. Foraminiferal Oxygen and Carbon stable isotope plots for Site U1490. Colored symbols indicate planktonic foraminifera, the black asterisk indicates benthic foraminifera. 

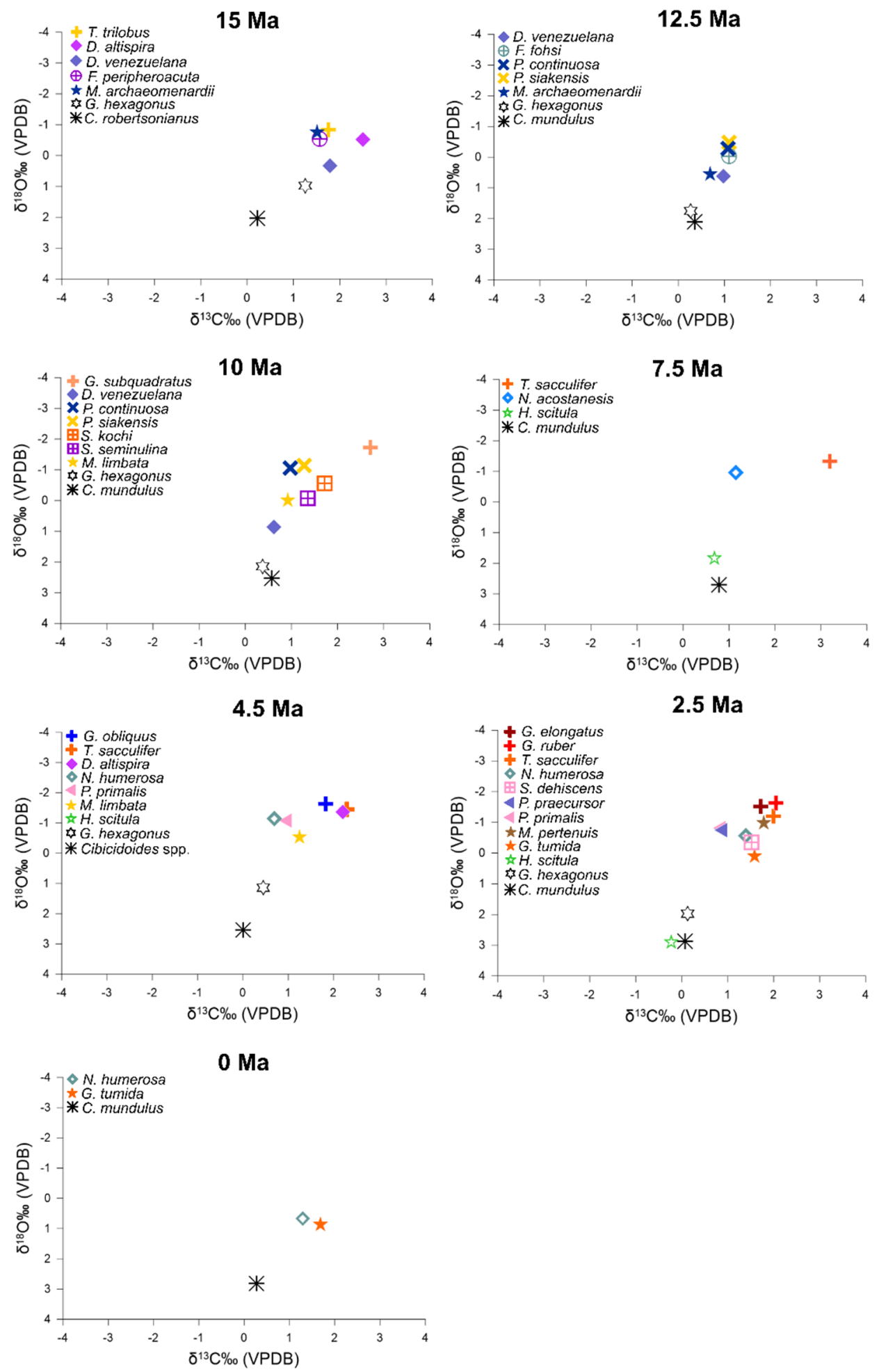

Figure S8. Foraminiferal Oxygen and Carbon stable isotope plots for Site U1338. Colored symbols indicate planktonic foraminifera, the black asterisk indicates benthic foraminifera. 

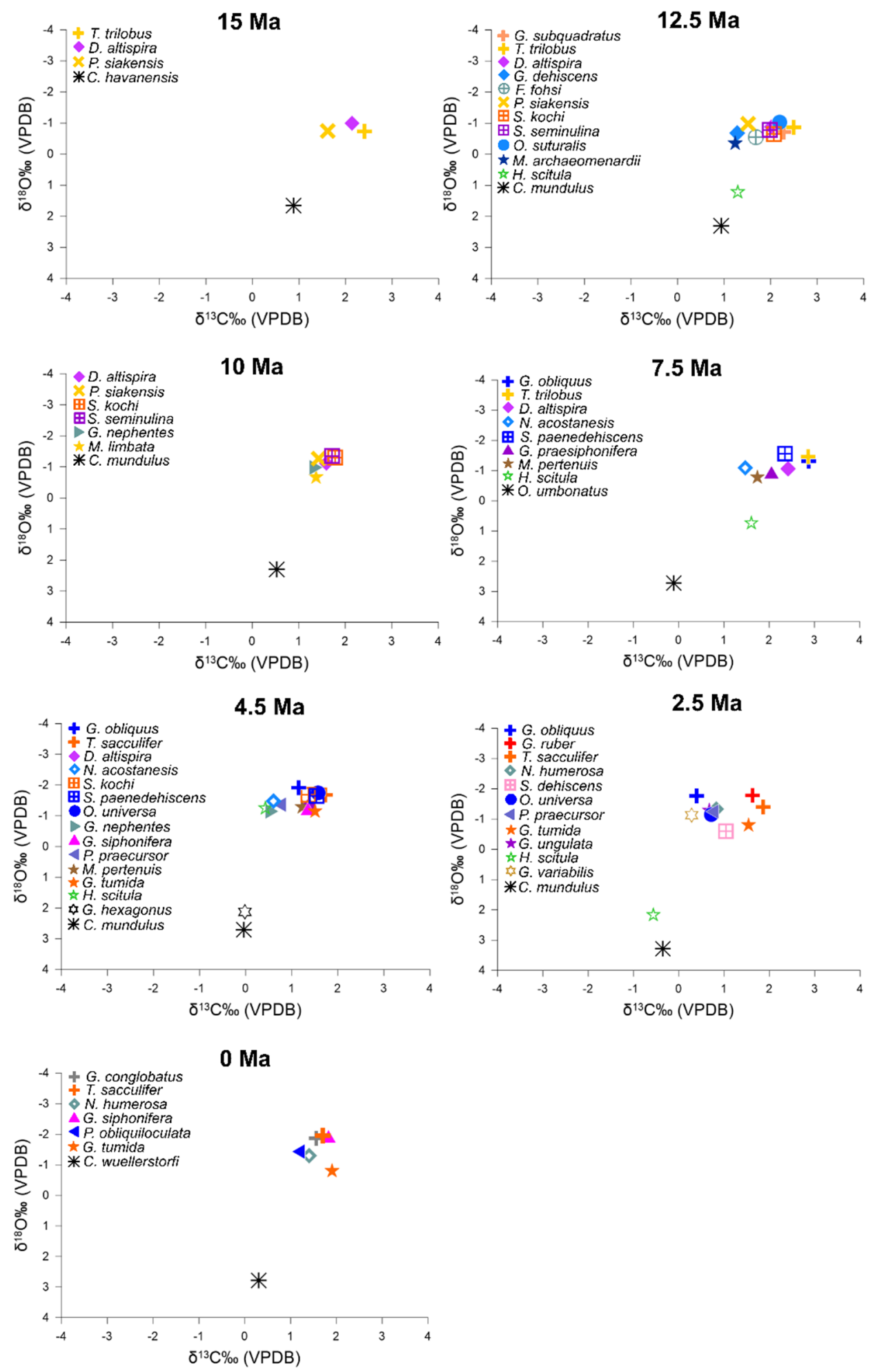

Figure S9. Foraminiferal Oxygen and Carbon stable isotope plots for Site U1489. Colored symbols indicate planktonic foraminifera, the black asterisk indicates benthic foraminifera. 
Table S1.

\begin{tabular}{|c|c|c|}
\hline & Foraminifera & Nannofossils \\
\hline Age & Site 871-872 & Site 871-872 \\
\hline $15 \mathrm{Ma}$ & $871 \mathrm{~A}-8 \mathrm{H}-1-\mathrm{W} 0 / 1 \mathrm{~cm}$ & $871 \mathrm{~A}-8 \mathrm{H}-1-\mathrm{W} 0 / 1 \mathrm{~cm}$ \\
\hline $12.5 \mathrm{Ma}$ & $871 \mathrm{~B}-5 \mathrm{H}-4-\mathrm{A} \quad 10 / 12 \mathrm{~cm}$ & $871 \mathrm{~B} 5 \mathrm{H}-1$ 12/14cm \\
\hline $10 \mathrm{Ma}$ & 872C-5H-4-W 83/85 cm & $872 \mathrm{C} 5 \mathrm{H}-4-\mathrm{W} 83 / 85 \mathrm{~cm}$ \\
\hline $4.5 \mathrm{Ma}$ & 872C-3H-5-W 116/118 cm & $872 \mathrm{C} 3 \mathrm{H}-5-\mathrm{W} 113 / 115 \mathrm{~cm}$ \\
\hline $2.5 \mathrm{Ma}$ & $872 \mathrm{~A}-2 \mathrm{H}-2-\mathrm{W} 120 / 121 \mathrm{~cm}$ & 872A 2H-2-W 120/121cm \\
\hline $0 \mathrm{Ma}$ & $872 \mathrm{~A}-1 \mathrm{H}-1-\mathrm{W} 0 / 2 \mathrm{~cm}$ & $\mathrm{X}$ \\
\hline Age & Site 242 & Site 242 \\
\hline $15 \mathrm{Ma}$ & 242Z-7R-6-W 145/147 cm & 242 7R-6-W 144/145cm \\
\hline $12.5 \mathrm{Ma}$ & 242Z-7R-6-W 13/15 cm & 242 7R-6-W 13/15cm \\
\hline $7.5 \mathrm{Ma}$ & 242Z-6R-3-W 3/5 cm & $2426 \mathrm{R}-3-\mathrm{W} 3 / 5 \mathrm{~cm}$ \\
\hline $4.5 \mathrm{Ma}$ & 242Z-3R-1-W 100/102 cm & 242 3R-1-W 100/102cm \\
\hline $2.5 \mathrm{Ma}$ & $242 \mathrm{Z}-2 \mathrm{R}-3-\mathrm{W} 3 / 5 \mathrm{~cm}$ & 242 2R-3-W 50/51cm \\
\hline $0 \mathrm{Ma}$ & 242Z-1R-2-W 45/47 cm & 242 1R-2-W 143/144cm \\
\hline Age & Site 1138 & Site 1138 \\
\hline $15 \mathrm{Ma}$ & U1138A-22R-1-W 20/22 cm & 1138A 22R-1-W 19/20cm \\
\hline $12.5 \mathrm{Ma}$ & U1138A-20R-1-W 40/42 cm & 1138A 20R-1-W 40/42cm \\
\hline $10 \mathrm{Ma}$ & U1138A-16R-2-W 30/32 cm & 1138A 16R-2-W 30/32cm \\
\hline $7.5 \mathrm{Ma}$ & U1138A-14R-2-W 140/142 cm & 1138A 14R-2-W 140/142cm \\
\hline $4.5 \mathrm{Ma}$ & U1138A-11R-5-W 130/132 cm & $\mathrm{X}$ \\
\hline $2.5 \mathrm{Ma}$ & U1138A-8R-3-W 130/132 cm & $\mathrm{X}$ \\
\hline $0 \mathrm{Ma}$ & U1138A-11R-1-W 0/2 cm & $\mathrm{X}$ \\
\hline Age & Site 516 & Site 516 \\
\hline $15 \mathrm{Ma}$ & $516 \mathrm{Z}-17 \mathrm{H}-4-\mathrm{W} \mathrm{0/2 \textrm {cm }}$ & $516 \mathrm{Z} 17 \mathrm{H}-4-\mathrm{W} 2 / 3.5 \mathrm{~cm}$ \\
\hline $7.5 \mathrm{Ma}$ & 516Z-14H-1-W 70/72 cm & $516 \mathrm{Z} 14 \mathrm{H}-1-\mathrm{W} 71 / 72.5 \mathrm{~cm}$ \\
\hline $4.5 \mathrm{Ma}$ & $516 Z-8 H-2-W 10 / 12 \mathrm{~cm}$ & $516 \mathrm{Z} 8 \mathrm{H}-2-\mathrm{W} 8 / 9.5 \mathrm{~cm}$ \\
\hline $2.5 \mathrm{Ma}$ & 516Z-3H-3-W 35-39 cm & $516 \mathrm{Z} 3 \mathrm{H}-3-\mathrm{W} 38 / 40 \mathrm{~cm}$ \\
\hline $0 \mathrm{Ma}$ & 516A-1H-1-W 50-52 cm & $516 \mathrm{Z} 1 \mathrm{H}-1-\mathrm{W} 50 / 52 \mathrm{~cm}$ \\
\hline Age & Site 1338 & Site 1338 \\
\hline $15 \mathrm{Ma}$ & U1338B-38H-4-W 52/54 cm & 1338B 38H-4-W 52/54cm \\
\hline $12.5 \mathrm{Ma}$ & U1338B-32H-3-W 67/69 cm & 1338B 32H-3-W 67/69cm \\
\hline $10 \mathrm{Ma}$ & U1338A-25H-6-W 66/68 cm & 1338A 25H-6-W 66/68cm \\
\hline $7.5 \mathrm{Ma}$ & U1338B-17H-4-W 12/14 cm & 1338B 17H-4-W 12/14cm \\
\hline $4.5 \mathrm{Ma}$ & $\mathrm{U} 1338 \mathrm{C}-7 \mathrm{H}-4-\mathrm{W}$ 60/62 cm & $1338 \mathrm{C} 7 \mathrm{H}-4-\mathrm{W} 60 / 62 \mathrm{~cm}$ \\
\hline $2.5 \mathrm{Ma}$ & U1338C-4H-4-W 60/62 cm & $1338 \mathrm{C} 4 \mathrm{H}-4-\mathrm{W} 60 / 62 \mathrm{~cm}$ \\
\hline $0 \mathrm{Ma}$ & U1338B-1H-1-W 3/5 cm & $1338 \mathrm{~B} 1 \mathrm{H}-1-\mathrm{W} 5 / 7 \mathrm{~cm}$ \\
\hline
\end{tabular}




\begin{tabular}{ccc}
\hline Age & Site 1489 & Site 1489 \\
\hline $15 \mathrm{Ma}$ & $\mathrm{U} 148941-2,22 / 24 \mathrm{~cm}$ & $\mathrm{X}$ \\
$12.5 \mathrm{Ma}$ & $\mathrm{U} 148938 \mathrm{X}-3,25 / 27 \mathrm{~cm}$ & $1489 \mathrm{C} 38 \mathrm{X}-3,25 / 27 \mathrm{~cm}$ \\
$10 \mathrm{Ma}$ & $\mathrm{U} 148933 \mathrm{X}-2,104 / 106 \mathrm{~cm}$ & $1489 \mathrm{C} 33 \mathrm{X}-2,104 / 106 \mathrm{~cm}$ \\
$7.5 \mathrm{Ma}$ & $\mathrm{U} 148924 \mathrm{H}-4,15 / 17 \mathrm{~cm}$ & $1489 \mathrm{C} 25 \mathrm{H}-4,15 / 17 \mathrm{~cm}$ \\
$4.5 \mathrm{Ma}$ & $\mathrm{U} 148912 \mathrm{H}-1,5 / 7 \mathrm{~cm}$ & $1489 \mathrm{~B} 12 \mathrm{H}-1,5 / 7 \mathrm{~cm}$ \\
$2.5 \mathrm{Ma}$ & $\mathrm{U} 14896 \mathrm{H}-6,85 / 87 \mathrm{~cm}$ & $1489 \mathrm{~B} 6 \mathrm{H}-6,85 / 87 \mathrm{~cm}$ \\
$0 \mathrm{Ma}$ & $\mathrm{U} 14891 \mathrm{H}-1,5 / 7 \mathrm{~cm}$ & $1489 \mathrm{~B} 1 \mathrm{H}-1,5 / 7 \mathrm{~cm}$ \\
\hline Age & Site $\mathbf{1 4 8 2}$ & Site 1482 \\
\hline $7.5 \mathrm{Ma}$ & $\mathrm{U} 148240 \mathrm{~F}-1,106 / 108 \mathrm{~cm}$ & $1482 \mathrm{~A} 40 \mathrm{~F}-1,106 / 108 \mathrm{~cm}$ \\
$4.5 \mathrm{Ma}$ & $\mathrm{U} 148224 \mathrm{H}-6,5 / 7 \mathrm{~cm}$ & $1482 \mathrm{~A} 24 \mathrm{H}-6,5 / 7 \mathrm{~cm}$ \\
$2.5 \mathrm{Ma}$ & $\mathrm{U} 148211-2,105 / 107 \mathrm{~cm}$ & $1482 \mathrm{~A} 11 \mathrm{H}-2,105 / 107 \mathrm{~cm}$ \\
$0 \mathrm{Ma}$ & $\mathrm{U} 14821 \mathrm{H}-1,7 / 9 \mathrm{~cm}$ & Site $\mathbf{1 4 9 0}$ \\
\hline Age & Site 1490 & $1490 \mathrm{~B} 26 \mathrm{~F}-1,45 / 47 \mathrm{~cm}$ \\
\hline $15 \mathrm{Ma}$ & $\mathrm{U} 149026 \mathrm{~F}-1,45 / 47 \mathrm{~cm}$ & $\mathrm{X}$ \\
$12.5 \mathrm{Ma}$ & $\mathrm{U} 149023-5,5 / 7 \mathrm{~cm}$ & $1490 \mathrm{C} 18 \mathrm{H}-2,45 / 47 \mathrm{~cm}$ \\
$7.5 \mathrm{Ma}$ & $\mathrm{U} 149018 \mathrm{H}-2,45 / 47 \mathrm{~cm}$ & $1490 \mathrm{~A} 10 \mathrm{H}-2,5 / 7 \mathrm{~cm}$ \\
$4.5 \mathrm{Ma}$ & $\mathrm{U} 149010 \mathrm{H}-2,5 / 7 \mathrm{~cm}$ & $1490 \mathrm{~A} 4 \mathrm{H}-7,35 / 37 \mathrm{~cm}$ \\
$2.5 \mathrm{Ma}$ & $\mathrm{U} 14904 \mathrm{H}-7,35 / 37 \mathrm{~cm}$ & $1490 \mathrm{~A} 1 \mathrm{H}-1,5 / 7 \mathrm{~cm}$ \\
$0 \mathrm{Ma}$ & $\mathrm{U} 14901 \mathrm{H}-1,5 / 7 \mathrm{~cm}$ & \\
\hline & &
\end{tabular}

